\title{
Evidence of between-population differences in natural selection on extrafloral nectaries of the shrub Anemopaegma album (Bignoniaceae).
}

\begin{tabular}{|r|l|}
\hline Journal: & Botany \\
\hline Manuscript ID & cjb-2015-0201.R2 \\
\hline Manuscript Type: & Article \\
\hline Date Submitted by the Author: & 26-Dec-2015 \\
\hline Complete List of Authors: & $\begin{array}{l}\text { Nogueira, Anselmo; University of São Paulo, Department of Botany } \\
\text { Rey, Pedro; Universidad de Jaén } \\
\text { Alcántara, Julio; Universidad de Jaén } \\
\text { Lohmann, Lúcia; University of São Paulo, Department of Botany }\end{array}$ \\
\hline Keyword: & $\begin{array}{l}\text { Adaptation, Adaptive landscape, Animal-plant interactions, Defensive traits, } \\
\text { Geographic mosaic }\end{array}$ \\
\hline &
\end{tabular}




\section{Evidence of between-population differences in natural selection on extrafloral nectaries of the shrub Anemopaegma album (Bignoniaceae).}

Anselmo Nogueira $^{1 *}$, Pedro J. Rey ${ }^{2}$, Julio M. Alcántara $^{2}$ \& Lúcia G. Lohmann ${ }^{1}$

${ }^{1}$ Universidade de São Paulo, Instituto de Biociências, Departamento de Botânica, Rua do Matão, 277, São PauloSP, 05508-090, Brazil.

${ }^{2}$ Universidad de Jaén, Facultad de Ciencias Experimentales, Departamento de Biología Animal, Biología

Vegetal y Ecología, Paraje Las Lagunillas s/n, 23071, Jaén, Spain.

*Corresponding author: +55 1198602 2566; anselmoeco@yahoo.com.br 


\section{Abstract}

Extra-floral nectaries (EFNs) are thought to represent adaptations against herbivores, but studies on the evolutionary ecology of EFNs have rarely been conducted. Here we investigate the patterns of natural selection and genetic variation on EFN traits in two wild populations of Anemopaegma album (Bignoniaceae) previously described as contrasting EFN-ant adapted localities on the Neotropical savanna. In each population four EFN descriptors, foliar damage and reproductive success variables were measured per plant (100-120 plants per population). To estimate the heritability of EFN traits we crossed reproductive plants in the field, and grew offspring plants in a common garden. Results showed that ant assemblages were different between populations as was the range of foliar herbivory. Genetic variation and positive phenotypic selection in EFN abundance was detected only in the Cristália population, in which plants with more EFNs were more likely to reproduce. An evaluation of putative causal links conducted by path analysis corroborated the existence of phenotypic selection on EFNs mediated by the herbivory process in the Cristália population. While EFNs could be currently under selection in Cristália, it is possible that past selection may have driven EFN traits to become locally adapted to the local ant assemblage in the Grão Mogol population.

Key-words: Adaptation; Adaptive landscape; Animal-plant interactions; Brazil; Defensive traits; Geographic mosaic; Geographic variation; Heritability; Phenotypic divergence; Plant-herbivore interactions. 


\section{Introduction}

The defensive action of plant-visiting ants is a classic example of mutualistic interaction modifying plant resistance against herbivores (Janzen 1966). This kind of association varies from obligatory and specialized (e.g., domatia offering a safe nest to ants) to facultative and less specialized interactions (e.g., extrafloral nectaries offering energetic resources to ants), and has been described for many plant species in distinct ecological conditions, demonstrating the generality of such associations (Bronstein et al. 2006; Rico-Gray and Oliveira 2007). In particular, much effort has been devoted to understanding the ecological role of extrafloral nectaries (EFNs) related to the potential negative effect of ant-EFN associations on herbivores (Rico-Gray and Oliveira 2007), and few studies have focused on the evolutionary ecology of EFNs and their interactions (e.g., Rutter and Rausher 2004). From a macro evolutionary perspective, clades with extrafloral nectaries diversified, in general, faster than lineages without them, probably due to the decrease of the extinction risk and increase of the opportunity for speciation through an expanded realized niche (Marazzi and Sanderson 2010; Weber and Agrawal 2014), which is in agreement with the hypothesis of herbivore avoidance. This pattern also agrees with comparative studies suggesting an adaptive role of EFN traits (e.g., Nogueira et al. 2012b) and the homoplastic pattern of EFN appearance among distinct and unrelated clades of angiosperms (Weber and Keeler 2013).

Under the perspective of the ecological theory of adaptive radiation (Schluter 2000), patterns observed at macroevolutionary scales should result from microevolutionary processes that are associated with different adaptive responses of plant populations to the action of natural selection on EFNs. Indeed, plant performance can vary among individuals within populations as a function of EFN trait variation (e.g., Rudgers 2004). This is due to the fact that the presence of ants mediated by EFNs decreases the probability of loss of plant tissue by herbivores while increasing the probability of plant reproduction (Rosumek et al. 2009; Trager et al. 2010). Studies of this nature have led to a general acceptance that ant-EFN plant defense have resulted from natural selection, despite the very limited data available on the heritability and phenotypic selection of EFN traits (Mitchell, 2004). Additionally, the intricate process of coevolution/evolution between associated organisms appears to occur gradually and in a highly variable way across populations (Thompson 2005, 2013), highlighting the necessity of additional studies on EFN evolution among populations of the same species.

Ant-plant mutualisms are conditional or context dependent (Bronstein 1994; Chamberlain and Holland 2009; Baker-Méio and Marquis 2012; Manzaneda and Rey 2012). Therefore, phenotypic selection on EFNs and the evolutionary outcome of these interactions are expected to vary among populations of the same plant species 
according to the geographic variation in herbivore and ant visitor assemblages, added to other biotic and abiotic factors (Rudgers and Gardener 2004). The geographic variation in selection (selection mosaics) and in the evolutionary outcome of interactions have been suggested as a fundamental component in the co-evolutionary process between interacting species (Thompson 2005), and have been thought to be involved in plant trait evolution in response to interacting mutualistic animals (Rey et al. 2009). Nonetheless, few studies to date have explored the geographic or among-population variation in selection on EFNs and in its evolutionary outcome (see Rudgers and Strauss 2004).

Here we investigate the potential response of selection on EFNs in two wild and undisturbed populations of Anemopaegma album Mart. ex DC. (Bignoniaceae), a shrub distributed throughout the Neotropical savannas of South America. These two A. album populations were chosen in the field among 10 populations previously investigated in Nogueira et al. (2015) because of their contrasting eco-evolutionary pattern of ant-plant-herbivore interactions. These two populations differ in (i) the functional relationship between phenotypic traits of plants and ants, (ii) mean levels of herbivory and plant performance, and (iii) EFN trait variation within population. While the first population (Grão Mogol) was previously described as matched population with higher mean plant performance and narrower variance of EFN abundance - a plant evolutionary hotspot (i.e., a population with ant-adapted EFNs); the second population (Cristália) was mismatched, with lower performance and larger variance of EFN abundance - a plant evolutionary coldspot (i.e., a population with mal-adapted EFNs). Our hypothesis is that due to the past natural selection the population with ant-adapted EFNs would be less sensitive to selection pressure in the present. Overall, we determine the potential of these two populations to adaptively respond to selection pressures exerted by ants and herbivores by addressing four main questions: (i) Is there preexisting variation in EFN traits among individuals and populations? (ii) Are some EFN traits heritable? (iii) Does phenotypic selection on EFN traits occur under current ecological conditions? (iv) Are the relationships between EFN traits, herbivory, and plant performance in accordance with the defensive hypothesis of EFN-ant selection against herbivores?

\section{Methods}

\section{Study system}

This study was conducted at Grão Mogol State Park and surroundings, ca. $550 \mathrm{~km}$ north of Belo Horizonte (Minas Gerais, Brazil). Further details about the soil and climatic conditions of the study site are described in Nogueira et al. (2012a). The focal species Anemopaegma album (Bignoniaceae) has extrafloral 
nectaries on the leaflets (Figure 1A-C), and the anatomical variation, number, size, distribution, and nectar secretion pattern of those nectaries were described in Nogueira et al. (2012a, 2015).

The two populations of A.album used here (Grão Mogol and Cristália) were separated by 50 km from each other (for more details see Nogueira et al. 2015). Each population had ca. 100-120 mature plants marked and geo-referenced (reproductive individuals generally higher than $50 \mathrm{~cm}$ ) selected systematically along three parallels trails that were separated by fifty meters from each other. We included the maximum number of mature plants in the whole extension of each population in a wide geographical area $\left(\approx 20.000 \mathrm{~m}^{2}\right)$, in which plants were at least $5 \mathrm{~m}$ between each other.

\section{Plant features, herbivory and biotic interactions}

EFN traits, foliar herbivory and plant reproduction were estimated for each plant within the two selected A. album populations. Reproductive data from each individual plant (Figure 1D-E) was quantified in the field in four distinct moments during the rainy season (November 2009 to Apr 2010). In the last sampling, 15-20\% of leaflets per plant (30-90 leaflets per plant) were collected and subsequently used in the laboratory to describe the EFN variation and foliar herbivory among plants.

EFN traits were quantified in 6-9 leaflets per plant using a stereomicroscope, looking at different positions on the leaflets (details in Figure 2). On average, more than $90 \%$ of EFNs were clustered at the base of the abaxial surface of leaflets (Character 1 in Figure 2); therefore, EFN abundance at this position was considered our best EFN trait descriptor functionally related with the sugar-secretion pattern and ant visitation. We also measured the diameter of the secretory head of the largest nectary at the EFN cluster, which was used as an additional EFN descriptor (Figure 2).

Herbivory was estimated in the 15-20\% leaflets collected per plant by quantifying the total leaf area and damaged area on each leaflet by visual inspection, aided by an acrylic sheet with 0.3 and $0.4 \mathrm{~mm}^{2}$ grids. Herbivory was estimated as the proportion of the total leaf area that was removed from the sampled leaflets. Ant and herbivore censuses were performed in a subsample of 30 individual plants in each population in two different periods, $t_{0}$ and $t_{l}$ (early and late summer). In each period all ants and herbivores were sampled per plant in 15 min censuses conducted between 7 A.M. and 11.30 A.M., and between 2.00 P.M and 6.30 P.M (herbivores that feed on A.album are mainly active in daytime), avoiding the highest temperatures at midday. Specimens of ant and herbivore morphotypes were collected and fixed in $90 \%$ alcohol for subsequent identification. The average number of insects per plant (standardized by the number of leaves) was calculated in order to 
characterize the variation of ant and herbivore assemblages in each population. We also looked at the ant and herbivore behaviors on plants to confirm the EFN use by ant species and the plant tissue damages by herbivores.

The variation of plant reproduction among individuals in each population was estimated based on four distinct sampling moments assessing the number of flowers and fruits per plant. Each assessment was separated temporally per 28-45 days during the reproductive period, and not included precisely the flower or fruit production per plant especially in individuals with higher flower production (personal observation during each sampling moment). Additionally, a great number of plants (around $50 \%$ of plants) didn't produce any flower or fruit in both populations. Therefore, based on these particularities of our study system we used a binary response variable to score the reproductive success of individual plants: 0 for total reproductive failure, and 1 for plants that achieve any positive production of flowers and fruits.

\section{Experimental plant crossings}

To estimate the heritability of EFN traits we crossed reproductive plants in the field, and grew offspring plants that resulted from such crosses in a greenhouse with controlled environmental condition. Previous tests in the field showed that $A$. album is self-incompatible (data not shown). In each population, 50 reproductive plants were used for the cross-pollination experiments. Among the 50 sampled plants within each population, 25 were treated as sire plants (i.e., plants that donate pollen), and the other 25 were treated as dam plants (i.e., receptors of pollen grains), totaling 25 independent experimental crossings $(\mathrm{N}=25)$ for each population. First, five to ten pre-anthesis flower buds of each plant were isolated from floral visitors with bridal veil bags. On the second day of anthesis, we pollinated the flowers using thin disposable sticks. Flowers were bagged again immediately after the manual pollination and monitored until fruit development or flower abscission.

Flowering period within and among plants spread along several weeks; crossings were thus performed sequentially until completing 25 crossings for each population. Fortunately, the flowering period of plants overlapped extensively, so we could avoid the potential problems of assortative mating. A large number of experimental crossings failed to set fruits or to produce seedlings afterwards, diminishing the number of independent families by population to 7 in Cristália and 14 in Grão Mogol. The experimental fruits obtained were sundried and the seeds brought back to the Institute of Biosciences of the University of São Paulo (São Paulo, Brazil) (Figure 1F). Each experimental fruit produced 30 seeds on average, giving rise to a family of at least 10 seedlings that were grown in the greenhouse under standardized conditions of solar radiation, irrigation, soil and biotic factors (Figure 1G-I). Plantings were conducted between November and December 2010, and the 
seeds were arranged inside a greenhouse in blocks of 15 to 25 pots. To avoid the effects of non-genetic factors that could increase trait similarity among offspring from the same family, seeds from the same experimental family were sowed only once in each block within the greenhouse. The location of each block within the greenhouse was randomized before its establishment. This arrangement was adopted to avoid the undesirable effect of unexpected or imperceptible environmental gradients within the greenhouse. This procedure was followed independently for each A. album population.

Because woody plant species from Neotropical savannas frequently have long and complex subterranean systems (Gottsberger and Silberbauer-Gottsberger 2006), we used PVC tubes with $1.20 \mathrm{~m}$ in height and $0.15 \mathrm{~cm}$ in diameter (Figure 1F, G and I) for seed planting, preventing radicular restriction during plant growth. Growing conditions included standardized soil (Tropstrato, Vida Verde), regular irrigation within the greenhouse at least twice/day, high irradiance, high nutritional conditions and the exclusion of insects. These conditions aimed at providing the most complete resources for plant development as possible. Sampling of EFNs on leaflets was conducted between April and May 2013, after the first plants produced flowers inside the greenhouse (Figure 1I), which was interpreted as evidence for plant maturation.

\section{Data analysis}

ANOVA and ANCOVA were performed to describe the variation of EFN traits and herbivory between the two populations. Normality and homoscedasticity were checked in all analyses, and normalizing transformations were applied in some cases as indicated in the results. If the transformations were not effective (e.g. normalizing the original data) we performed linear regressions by permutation process.

Trait heritability was estimated from the degree of resemblance between relatives (Falconer and Mackay 1996). In our estimates, we applied a special case of offspring-parent regression, in which the $h_{\text {min }}^{2}$ (lower bound heritability in a natural environment) was estimated for each plant character using field and labreared plants per population (see details in Riska et al. 1989; and Lynch and Walsh 1998). First, we regressed the dataset of the parents growing in the field (mid-parent) to the lab-reared sibs growing in the common garden (mid-offspring), and subsequently applied an approximation to calculate the $h^{2}{ }_{\text {min }}$ following Riska et al. (1989). The lower bound heritability $\left(h_{\text {min }}^{2}\right)$ is conservative because the estimate of additive genetic variance from the greenhouse, which was used to calculate the lower bound, can be inflated by dominance, common environment or maternal effects (Riska et al. 1989). We also performed classical dam and sire-offspring regressions to explore 
potential maternal effects on EFN trait variation in the offspring, inferred by the difference between regression models (dam and sire) in each population.

We applied logistic regression to estimate the strength of selection on EFN traits in each population of A. album. This approach is more suitable than linear regression for analyzing data from selection studies with dichotomous fitness outcomes (for example 0 and 1 for plant reproduction). The estimated logistic coefficient was transformed to values of the estimated selection gradient, or average gradient of selection surface $-\beta_{\text {avggrad }}+$, following the procedure described by Janzen and Stern (1998).

The general causal model according to the hypothesis of defense mediated by extrafloral nectaries predicts that nectaries attract ants that deter herbivores, decreasing herbivory and increasing plant reproduction. Considering the two main descriptors of extrafloral nectaries (i.e., EFN abundance on the leaflet base and EFN size), herbivory and plant reproduction, we used an alternative confirmatory path analysis to test for the defense hypothesis in each population separately. This analysis consisted of a generalization of Shipley's d-sep test that could be used on variables having different sampling distribution (Shipley 2009) as encountered in our study system (e.g., plant reproduction described as a binary variable). Path analyses were performed in five steps as suggested by Shipley (2009): (i) construction of hypothetical causal relationships between variables in the form of directed acyclic graph; (ii) list of each pair of variables without a direct link on the acyclic graph; (iii) definition of a full set of the $k$ independence claims [i.e. $(X i, X j) \mid\{Z\}]$ that formed the basis set $\mathrm{B}_{\mathrm{U}}$; (iv) for each element in this basis set, the probability, $p_{k}$, was obtained so that the pairs [i.e. $\left.(X i, X j)\right]$ were statistically independent conditionals on the variables $\{\mathrm{Z}\}$; (v) the k probabilities $\left(p_{i}\right)$ were combined using the $C$ equation. Finally, the $C$ value was compared to a chi-squared distribution with $2 k$ degrees of freedom. We rejected the causal model whenever the $C$ value was unlikely to have occurred by chance (below the chosen significance level). The $C$ equation proposed by Shipley is $C=-2 \sum_{i=1}^{k} \ln (p i)$. After confirming or rejecting the hypothetical causal model, the path coefficients were obtained by regressing each variable within each of its direct causes. This approach is largely detailed in Shipley (2009).

Statistical analyses (e.g., ANOVA, ANCOVA, logistic regression and path analyses) were performed in R v. 3.0.1 (R Development Core Team 2009) using standard package, and linear regression with permutation tests was performed using the $1 \mathrm{mPerm}$ package. 


\section{Results}

\section{Ecological and phenotypic scenario in the studied populations}

Plants from Grão Mogol on average had $16 \%$ more EFNs at the base of leaflets; in addition, nectaries were $3.6 \%$ larger than those from Cristália (Figure 3), but EFNs scattered in the leaf blades did not differ significantly between populations (Supplementary Table S1). In the Grão Mogol population, 51.7 \% of the plants flowered, while only $38.1 \%$ of the plants flowered in Cristália. Additionally, reproductive plants from Grão Mogol produced on average three times more flowers (Supplementary Table S1) and 2.7 times more fruits than Cristália (data not shown).

The average abundance of ants per plant was greater in Cristália than in Grão Mogol $\left(\mathrm{F}_{(1,60)}=4.98 ; \mathrm{p}=\right.$ 0.029; results with ant abundance log-transformed) (Figure 4A), and the number of ant species per plant was also greater in Cristália $\left(\mathrm{F}_{(1,60)}=6.47 ; \mathrm{p}=0.011\right.$; modeling with the Poisson distribution). The potential defensive role of ant species was more variable among plants in the Cristália than in Grão Mogol population (Figure 4B-D). For both populations the potential protective role of each ant species were scored based on the herbivory index (Nogueira et al. 2015, Figure 4B), in which the Cristália population had around $50 \%$ of plants occupied by less or non protective ants (e.g. Pseudomyrmex gracilis and Cephalotes pusillus - Figure 4C), and the remaining plants occupied by more protective ants (e.g. Camponotus crassus). Differently, Grão Mogol population had most plants occupied by more protective ant species (e.g. Camponotus blandus and Camponotus novogranadensis - Figure 4D).

Plants in Cristália showed a wider range of herbivory (0.1 to 100\%) than plants in Grão Mogol (1 to $63.8 \%$ ), but their population means did not differ significantly (Supplementary Table S1). The average abundance of herbivores per plant was similar in the two populations sampled $\left(\mathrm{F}_{(1,60)}=0.09 ; \mathrm{p}=0.77\right.$; results with herbivores abundance log-transformed), but when we considered the total number of herbivores summed across plants in each population, we observed 284 and 148 adult herbivores on plants of Cristália and Grão Mogol populations in the early summer, respectively. This large difference between populations (around two times) seems to disappear in the late summer, in which 119 and 114 adult herbivores were observed in these populations.

\section{Is EFN variation heritable?}

The lower bound for heritability in nature $\left(h_{\text {min }}^{2}\right)$ was estimated for all EFN traits in each population separately (Table 1). EFN abundance on the base of leaflets was heritable only in Cristália (value significantly 
greater than zero at the $5 \%$ level). Midparent-offspring and sire-offspring regressions of EFN abundance on the base of leaflets showed the same pattern of heritability in this population, suggesting negligible genetic maternal effects on EFN variation among plants (Supplementary Figure S1). In contrast, Grão Mogol population had a positive heritability only for the EFN abundance on the adaxial surface of leaflets (a leaflet region with just one EFN on average). The phenotypic variation of EFN abundance on the base of leaflets among individuals (the most important region of EFN variation due to the absolute number of EFN on the leaves) did not show any evidence of heritability in the Grão Mogol population (Table 1).

Additionally, EFN abundance was higher in the field plants (mid-parent) than in the common garden (mid-offspring) in both populations of A.album sampled (Supplementary Figure S1). On average, field plants had 3.3 and 2.4 times more nectaries than offspring plants in the Grão Mogol and Cristália populations, respectively.

\section{Is there phenotypic selection on EFN traits?}

Plants with a higher number of EFNs on the leaflets (on the base and the middle/apex of abaxial side) had a greater probability of reproduction in the Cristália population, indicating positive directional selection on EFN abundance in this population (Figure 5A and C; Table 2). In contrast, the Grão Mogol population did not show any evidence for phenotypic selection on EFN traits among individual plants (Figure 5; Table 2).

What is the mechanism behind evolutionary differences between populations?

The defensive hypothesis (causal model described above) was corroborated by path analyses only for the Cristália population (Figure 6; and Supplementary Table S2, S3 and Supplementary Figure S2 for details). In this population, the larger the nectaries, the lower the herbivory; and the negative relationship between the EFN abundance and herbivory was marginally significant (Figure 6A, Supplementary Table S3 and Supplementary Figure S2). In turn, the lesser herbivory the greater the probability of plant reproduction. In Grão Mogol, the causal model was completely rejected, and none of the four variables followed the expectations from the defense hypothesis mediated by extrafloral nectaries (Figure 6B, Supplementary Table S3 and Supplementary Figure S2). 


\section{Discussion}

Facultative interactions between ants and plants can be mediated by extrafloral nectaries that secrete a sugar-rich resource in exchange for potential ant-guard services against herbivores (references in Introduction), an opportunity for a mutualistic interaction between ants and plants. Sugar-secreting EFNs are thus widely thought to have resulted from natural selection and to represent plant adaptations that provide ant-mediated defense against insect herbivores. However, quantitative evaluation on phenotypic selection and the potential to respond to selection of EFN traits have rarely been conducted (Mitchell 2004). Here we evaluate the process of natural selection on EFN traits in two wild populations of $A$. album previously described as distinct ecoevolutionary localities: (i) the Grão Mogol population, a plant-evolutionary hot spot in which EFN traits optimize ant attraction and enhance plant reproductive success (EFN-ant adapted population), and (ii) the Cristália population, a cold spot in which EFN traits and its ant assemblage are not adapted to reduce herbivory (Nogueira et al. 2015). Here, we found evidence of phenotypic selection and heritability on EFNs just in the Cristália population (a cold spot population) in accordance with our previous hypothesis (Introduction).

Moreover, the EFN evolution as plant defense mediated by ant guarding was also corroborated in Cristália, but not in the Grão Mogol population. These results are here discussed in relation to the herbivore pressure and ant visitor assemblages, as well as in the context of the selection mosaics and geographic structure of the interaction described for this system.

\section{Natural selection on EFN traits: heritability and phenotypic selection}

At least two types of information or sub-processes are necessary to demonstrate that selective pressures can produce evolutionary change (response to selection) in plant populations: (i) trait heritability and (ii) differential reproduction mediated by the traits of interest (Endler 1986).

\section{Heritability of EFN traits}

To date, heritable EFN trait variation (i.e., genetic variance of EFN traits) has been reported in just two plant species: Chamaecrista fasciculata (Rutter and Rausher 2004) and Gossypium thurberi (Rudgers 2004). Here, we found heritable variation for some of the EFN traits in the two populations of Anemopaegma album. Although most EFN traits of $A$. album populations lacked heritable variation, our results must be taken with caution as small sample sizes may lead to an underestimation of the number of EFN traits that harbor heritable 
variation. In any case, the heritability of EFN traits in Grão Mogol had much smaller values than in Cristália even though the first had twice the sample size than the later.

Low levels of additive genetic variance of EFNs may severely condition the phenotypic change between generations through time (Templenton 2006). Low levels of genetic variance in a population are commonly interpreted as recent bottlenecks (or possible founder effects) or as a result of strong stabilizing or directional selection. In populations that are locally adapted to their biotic or abiotic agents of selection, directional or stabilizing selection working over generations would erode additive genetic variance. Even though we lack data on whether the Grão Mogol population may have been subject to recent bottlenecks (e.g., forest fires), Grão Mogol seems to represent a hot spot that is locally adapted to its ant assemblages (Nogueira et al. 2015). On the other hand, erosion of the genetic variance is not expected in localities in which the evolutionary adjustment between plant traits and ant visitor assemblages are far from equilibrium (cold spot), as in the Cristália population (Nogueira et al. 2015).

Non-adaptive mechanisms may also shape genetic and phenotypic variation within and among populations. Plant traits, including EFN traits, can also vary in response to non-selective environmental variation and a substantial amount of genetic variation may be swamped out in the field (Mitchell 2004). Maternal effects could be the first cause of such kind of phenotypic variation. However, in the Cristália population we detected a similar pattern of midparent-offspring regressions on EFN abundance with the sire-offspring regression, and not with the dam-offspring regression, which suggests lack of maternal effects on EFN trait variation. In agreement, maternal effects through seed size were not significant on EFN traits on plants of Chamaecrista fasciculata (Rios et al. 2008).

Phenotypic plasticity may also produce substantial trait variation in the field and such variation may be also adaptive (e.g. induced responses), which allows organisms to maximize fitness by optimizing the expression of a specific trait (Stearns 2014). Plasticity of EFN traits is commonly described as an inducible defensive response under high herbivore damage (Agrawal and Rutter 1998; Heil et al. 2001; Heil 2008). In this context, A. album plants with higher herbivory levels might be associated with larger production or expression of EFN traits, while the opposite pattern might be expected in plants grown in common garden with the exclusion of herbivores. This was actually the scenario encountered in our study, in which the parental field plants had higher EFN abundance than their offspring grown in the greenhouse. Nonetheless, the lower bound of heritability estimated using field parental and greenhouse offspring plants is thought to represent a better experimental procedure to measure heritability than estimates obtained from plants grown exclusively in the greenhouse 
(Riska et al. 1989). Such procedure has been previously used in the wild cotton Gossypium thurberi, in which low proportion of EFN offspring variance was attributable to the parents (sire-offspring regression in Rudgers 2004), a pattern that might be associated with the high degree of EFN trait plasticity. Although some phenotypic plasticity was present in our populations, we found a higher heritability for EFN trait variation (i.e., EFN abundance in the leaflet base in Cristália population) than that reported for Gossypium thurberi (Rudgers 2004).

\section{Phenotypic selection on EFN traits}

We detected a distinct phenotypic selection pattern between localities: (i) weak positive selection on EFN abundance in Cristália, and (ii) absence of selection in Grão Mogol, which provides further support to the idea that a mosaic selection hypothesis (sensu Thompson 2005) will frequently emerge in ant-guard plant defensive mutualisms. In fact, corroborating the commonness of selection mosaics in this particular animal-plant interaction, Rudgers and Strauss (2004) showed evidence of inter-population variation in EFN phenotypic selection and in its evolutionary outcome in other ant-guard system mediated by extrafloral nectaries. Positive selection on EFN abundance is an expected result when (i) populations are exposed to high levels of herbivore damage; and (ii) whenever most protective ant visitors of the local assemblage are attracted by higher density of EFNs. Plants of A. album have Camponotus ants as the most common defensive ant visitors, and those ants were attracted by the abundant EFNs located at the leaflet bases (Nogueira et al. 2015). Plants from Cristália had a lower EFN abundance; however, ants were more abundant and their species composition and defensive role were more variable among plants than in the Grão Mogol population. Indeed, Cristália had 50 \% of plants occupied by less- or non-protective ants, differently from Grão Mogol with most plants occupied by more protective ant species (a pattern independent of the average number of ants per plant in each population). Therefore, it is not surprising that ants might have exerted positive selection on EFNs, especially in the Cristália population, which is congruent with a higher variability between plants in herbivore damage (with some plants suffering extreme damages).

We cannot discard that the positive selection denoted by the relationship between the EFN traits and reproductive success in the Cristália population might also be caused by genetic correlation between unmeasured traits under selection. Unfortunately, we do not have any complementary evidence to be able to exclude this last hypothesis, which could be explored in further quantitative genetic studies.

What is the mechanism behind evolutionary differences between populations? 
The mechanism underlying differences among plant populations in phenotypic selection mediated by animal interactions has been commonly explored through structural equation modelling and path analysis (e.g., Gómez and Zamora 2000; Rey et al. 2006). We followed this approach to elaborate a model that aims to answer whether the relationships between EFN traits, herbivory, and plant performance at each population are in accordance with the hypothesis of EFN evolution as a defensive trait against herbivores promoted by ant visitation. We showed positive phenotypic selection on EFNs and demonstrated that the selection occurs through the defensive function of EFNs which, in turn, decreases herbivore damage and enhances plant reproductive success in the Cristália population, the only one in which phenotypic selection was verified. Through those results, we were able to demonstrate that positive phenotypic selection of EFNs is associated with increased leaf defense in the Cristália population while EFN variation is presently decoupled from variation in leaf defense in Grão Mogol, explaining the lack of selection in this population. Apparently, herbivore pressure (i.e., herbivore abundance per plant) was similar on the two populations, but the abundance of ants was clearly lower in Grão Mogol, which might lead to the variable pattern of selection encountered between localities.

\section{Plant adaptation: present-day versus past selection on extrafloral nectary traits}

Our results indicate that the actual conditions in Cristália allowed the adaptive change of EFNs of $A$. album: the ant assemblage and its potential protective role is variable among plants, and some common ant species (e.g. Camponotus crassus) that are attracted by abundant large nectaries in the base of leaflets (Nogueira et al. 2015) efficiently deter herbivores, enhancing plant reproductive success in some individuals within this population. However, the ant-guard-EFN system in this population is still far from equilibrium since considerable additive genetic variance remains in the EFN trait subjected to selection. In a comparison among ten A. album populations, Nogueira et al. (2015) showed that the Cristália population was among those with lowest plant performance, and the size and abundance of EFNs in the leaflets of its plants fall out of the trajectory predicted by the phenotypic interface between ants and EFNs (see Nogueira et al. 2015, for more details). All of these findings reinforce the idea that this population constitutes a plant evolutionary cold spot in the geographic structure and evolution of this plant-ant guard system.

In contrast, data from the Grão Mogol population suggest that this population may be at (or close to) an adaptive equilibrium with its ant visitor assemblage. In this population, it is possible that past selection may have driven EFN abundance and size to become locally adapted to its local ant assemblages. This hypothesis agrees with the lack of additive genetic variance and might explain the absence of EFN selection detected here, given 
that this population included a narrower range of phenotypes and plants with high reproductive performance. Opposite to what was observed in Cristália, the Grão Mogol population falls within the trajectory predicted by the phenotypic interface of the interaction for the local adjustment between partners (Nogueira et al. 2015). All of which were congruent with the hypothesis that this population represents a plant evolutionary hot spot of these interactions.

In sum, the two focal populations represent different present-day scenarios for natural selection and evolution of EFNs as defensive traits in A. album. Past evolutionary processes (e.g., differences in the heritability and population mean of EFNs) and present-day ant-plant interactions (e.g., differences of ant abundance, diversity and function) might explain the alternative evolutionary scenarios found in each population. Yet, questions still remain unsolved about the role of natural selection over time in this plant-ant guard system. The high ant abundance in tropical environments could indicate certain types of constancy in ant assemblages through time (e.g., Díaz-Castelazo et al. 2013), as opposed to from the herbivore assemblages that might vary strongly between years. The lack of long-term studies in which EFN-ant associations are monitored (e.g., more than five consecutive years) prevent any predictions about the constancy of selection through time, and should stimulate new long-term projects on animal-plant interactions in an eco-evolutionary perspective. However, the combination of natural selection analysis in focal populations together with the examination of geographical variation of ant-plant interactions and its evolutionary outcomes (phenotypic matching between partners) provides key insights into the evolutionary potential of EFN traits in particular plant species and populations (Rudgers and Strauss 2004; Rios et al. 2008; Nogueira et al. 2015). Future experimental and longterm research may elucidate the specific role of ants on herbivores and accurately describe the temporal variation of those interactions and plant outcomes across populations.

\section{Acknowledgements}

This project was funded by the Fundação de Amparo à Pesquisa do Estado de São Paulo (FAPESP Fellowships to A.N. 2012/02110-5 and A.N. 2013/04591-3, and FAPESP grant to L.G.L. 2007/55433-8), a Pq-1C grant from the CNPq to L.G.L. Logistic support was provided by IB-USP and the State Park of Grão Mogol (Minas Gerais/Brazil). We are gratefully to C.L. Silva-Luz, J.C. Lopes and E. Carbonell for assistance during fieldwork; R. Feitosa for identification of ant assemblages; and A.P.A. Assis, J.G. Rando, J.C. Friedman, L.H. Fonseca and M. Pace for fruitful discussions on the manuscript. We also thank K. Donohue and the reviewers at Axios 
Review for helping to improve the manuscript. Co-author emails:prey@ujaen.es (PRJ);jmalcan@ujaen.es (JMA); llohmann@usp.br(LGL).

\section{References}

Agrawal, A.A. and Rutter, M.T. 1998. Dynamic anti-herbivore defense in ant-plants: the role of induced responses. Oikos 83(2): 227-236. doi: 10.2307/3546834

Baker-Méio, B. and Marquis, R.J. 2012. Context-dependent benefits from ant-plant mutualism in three sympatric varieties of Chamaecrista desvauxii. J. Ecol. 100(1): 242-252. doi: 10.1111/j.1365-2745.2011.01892.x

Bronstein, J.L. 1994. Conditional outcomes in mutualistic interactions. Trends Ecol. Evol. 9(6): 214-217. doi: 10.1016/0169-5347(94)90246-1

Bronstein, J.L., Alarcón, R. and Geber, M. 2006. The evolution of plant-insect mutualisms. New Phytol. 172(3): 412-428. doi: 10.1111/j.1469-8137.2006.01864.x

Chamberlain, S.A. and Holland, J.N. 2009. Quantitative synthesis of context dependency in ant-plant protection mutualisms. Ecology 90(9): 2384-92. Available from http://www.jstor.org/stable/25592765

Díaz-Castelazo, C., Sánchez-Galván, I., Guimarães, P., Raimundo, R.L.G. and Rico-Gray, V. 2013. Long-term temporal variation in the organization of an ant-plant network. Ann. Bot. 111(6): 1285-1293. doi: $10.1093 / \mathrm{aob} / \mathrm{mct} 071$

Endler, J.A. 1986. Natural selection in the wild. Princeton University Press, Princeton, USA.

Falconer, D.S. and Mackay, T.F.C. 1996. Introduction to quantitative genetics. Longman Group ltd, London, UK.

Gómez, J. and Zamora, R. 2000. Spatial variation in the selective scenarios of Hormathophylla spinosa (Cruciferae). Am. Nat. 155(5): 657-668. doi: 10.1086/303353

Gottsberger, G. and Silberbauer-Gottsberger, I. 2006. Life in the cerrado: a south american tropical seasonal ecosystem. Vol. I Origin, Structure, Dynamics and Plant Use. Universitat Ulm, Ulm: Reta Verlag (Germany).

Heil, M., Koch, T., Hilpert, A., Fiala, B., Boland, W. and Linsenmair, K.E. 2001. Extrafloral nectar production of the ant-associated plant, Macaranga tanarius, is an induced, indirect, defensive response elicited by jasmonic acid. PNAS 98(3): 1083-8. doi: 10.1073/pnas.98.3.1083

Heil, M. 2008. Indirect defence via tritrophic interactions. New Phytol. 178(1): 41-61. doi: 10.1111/j.14698137.2007.02330.x

Janzen, D.H. 1966. Coevolution of mutualism between ants and acacias in Central America. Evolution 20(3): 249-275. Available from http://links.jstor.org/sici?sici=0014$3820 \% 28196609 \% 2920 \% 3$ A3\%3C249\%3ACOMBAA\%3E2.0.CO\%3B2-\%23

Janzen, F.J. and Stern, H.S. 1998. Logistic regression for empirical studies of multivariate selection. Evolution 52(6): 1564-1571. doi: 10.2307/2411330

Lynch, M. and Walsh, B. 1998. Genetics and analysis of quantitative traits. Sinauer Associates, Sunderland, USA. 
Manzaneda, A.J. and Rey, P.J. 2012. Geographical and interspecific variation and the nutrient-enrichment hypothesis as an adaptive advantage of myrmecochory. Ecography 35(4): 322-332. 10.1111/j.16000587.2011.06923.x

Marazzi, B. and Sanderson, M.J. 2010. Large-scale patterns of diversification in the widespread legume genus Senna and the evolutionary role of extrafloral nectaries. Evolution 64(12): 3570-3592. doi: 10.1111/j.1558-5646.2010.01086.x

Mitchell, R.J. 2004. Heritability of nectar traits: why do we know so little? Ecology 85(6): 1527-1533. doi: $\underline{10.1890 / 03-0388}$

Nogueira, A., Guimarães, E., Machado, S.R. and Lohmann, L.G. 2012a. Do extrafloral nectaries present a defensive role against herbivores in two species of the family Bignoniaceae in a Neotropical savanna? Plant Ecology 213(2): 289-301. doi: 10. 1007/ s11258-011-9974-3

Nogueira, A., Rey, P.J. and Lohmann, L.G. 2012b. Evolution of extrafloral nectaries: adaptive process and selective regime changes from forest to savanna. J. Evol. Biol. 25(11): 2325-40. doi: 10.1111/j.14209101.2012.02615.x.

Nogueira, A., Rey, P.J., Alcântara, J.M., Feitosa, R.M. and Lohmann, L.G. 2015. Geographic mosaic of plant evolution: extrafloral nectary variation mediated by ant and herbivore assemblages. PLoS ONE 10(4): e0123806. doi: 10.1371/journal.pone.0123806

R Development Core Team 2009. R: A language and environment for statistical computing. R Foundation for Statistical Computing. Vienna. Austria. Available from http://www.R-project.org.

Rey, P.J., Alcántara, J.M., Manzaneda, A.J., Garrido, J.L. and Ramírez, J.M. 2009. Variación geográfica y mosaicos de selección en las interacciones planta-animal. In R. Medel, R. Zamora, M. Aizen, and R. Dirzo (Eds.), Conservación de las interacciones planta-animal: conceptos y transferencias. pp. 113-132. Madrid/Santiago, España/Chile.

Rey, P.J., Herrera, C.M., Guitián, J., Cerdá, X., Sánchez-Lafuente, M., Medrano, M. and Garrido, J.L. 2006. The geographic mosaic in predispersal interactions and selection on Helleborus foetidus (Ranunculaceae). J. Evol. Biol. 19(1): 21-34. doi: 10.1111/j.1420-9101.2005.00992.x

Rico-Gray, V. and Oliveira, P.S. 2007. The ecology and evolution of ant-plant interactions. The University of Chicago Press, Chicago and London.

Rios, R.S., Marquis, R.J. and Flunker, J.C. 2008. Population variation in plant traits associated with ant attraction and herbivory in Chamaecrista fasciculata (Fabaceae). Oecologia 156(3): 577-88. doi: 10.1007/s00442-008-1024-Z.

Riska, B., Prout, T. and Turelli, M. 1989. Laboratory estimates of heritabilities and genetic correlations in nature. Genetics 123(4): 865-871. Available from http://www.ncbi.nlm.nih.gov/pmc/articles/PMC1203895/pdf/ge1234865.pdf

Rosumek, F.B., Silveira, F.A.O., Neves, F. de S., Barbosa, N.P. de U., Diniz, L., Oki, Y., Pezzini, F., Fernandes, G.W. and Cornelissen, T. 2009. Ants on plants: a meta-analysis of the role of ants as plant biotic defenses. Oecologia 160(3): 537-549. doi: 10. 1007/ s00442-009-1309-X

Rudgers, J.A. 2004. Enemies of herbivores can shape plant traits: selection in a facultative ant-plant mutualism. Ecology 85(1): 192-205. doi: $\underline{10.1890 / 02-0625}$ 
Rudgers, J.A. and Gardener, M.C. 2004. Extrafloral nectar as a resource mediating multispecies interactions. Ecology 85(6): 1495-1502. doi: 10.1890/03-0391

Rudgers, J.A. and Strauss, S.Y. 2004. A selection mosaic in the facultative mutualism between ants and wild cotton. Proc. R. Soc. Lon. 271(1556): 2481-2488. doi: 10.1098/rspb.2004.2900

Rutter, M.T. and Rausher, M.D. 2004. Natural selection on extrafloral nectar production in Chamaecrista fasciculata: the costs and benefits of a mutualism trait. Evolution 58(12): 2657-2668. Available from http://www.jstor.org/stable/3449421

Schluter, D. 2000. The ecology of adaptive radiation. Oxford Series in Ecology and Evolution, Oxford University Press, Oxford.

Shipley, B. 2009. Confirmatory path analysis in a generalized multilevel context. Ecology 90(2): 363-368. doi: $\underline{10.1890 / 08-1034.1}$

Stearns, S.C. 2014. Evolution of reaction norms. In J. B. Losos (Ed.), pp. 261-267. Princeton Guide to Evolution. Princeton University Press, New Jersey.

Templenton, A.R. 2006. Population genetics and microevolutionary theory. John Wiley \& Sons Inc., Hoboken, New Jersey.

Thompson, J.N. 2005. The geographic mosaic of coevolution. The University of Chicago Press, Chicago and London.

Thompson, J.N. 2013. Relentless evolution. The University of Chicago Press, Chicago and London.

Trager, M.D., Bhotika, S., Hostetler, J.A., Andrade, G.V., Rodriguez-Cabal, M.A., McKeon, C.S., Osenberg, C.W. and Bolker, B.M. 2010. Benefits for plants in ant-plant protective mutualisms: a meta-analysis. PLoS ONE 5(12): 1-9. doi: 10.1371/journal.pone.0014308

Weber, M.G. and Agrawal, A.A. 2014. Defense mutualisms enhance plant diversification. PNAS. 111(46): 16442-16447. doi: 10.1073/pnas.1413253111

Weber, M.G. and Keeler, K. 2013. The phylogenetic distribution of extrafloral nectaries in plants. Ann. Bot. 111(6): 1251-1261. doi: 10.1093/aob/mcs225. 


\section{Tables}

Table 1 - Parent-offspring regression analyses of EFN traits in A. album using parental midpoint from the field and mid-offspring from common garden. Analyses were also performed by jackknife procedures corroborating the heritability estimates for the EFN traits. Lower bound to the heritability in the field $\left(h^{2}{ }_{\text {min }}\right)$ and the confidence intervals (CI) were obtained as described by Riska et al. (1989) and Lynch \& Walsh (1998).

\begin{tabular}{|c|c|c|c|c|c|}
\hline \multirow{2}{*}{ Population } & \multirow{2}{*}{ EFN traits } & \multicolumn{3}{|c|}{ Midparent-offspring regression } & \multirow{2}{*}{$h_{\min }^{2} \pm \mathrm{CI}$} \\
\hline & & $B$ & SE & $p$ & \\
\hline $\mathrm{Cr}$ & 1 - EFN base & 0.30 & 0.12 & 0.049 & $0.24 \pm 0.095$ \\
\hline $\mathrm{Cr}$ & 2 - EFN size (mm) & 36.51 & 17.73 & 0.094 & $0.02 \pm 0.014$ \\
\hline $\mathrm{Cr}$ & 3 - EFN abaxial $\left(\mathrm{cm}^{2}\right)$ & 0.19 & 0.24 & 0.465 & $0.02 \pm 0.083$ \\
\hline $\mathrm{Cr}$ & 4 - EFN adaxial $\left(\mathrm{cm}^{2}\right)$ & 0.20 & 0.17 & 0.314 & $0.07 \pm 0.119$ \\
\hline $\mathrm{Gm}$ & 1 - EFN base & -0.07 & 0.14 & 0.620 & $0.001 \pm 0.076$ \\
\hline $\mathrm{Gm}$ & 2 - EFN size (mm) & 0.04 & 0.33 & 0.896 & $0.001 \pm 0.033$ \\
\hline $\mathrm{Gm}$ & 3- EFN abaxial $\left(\mathrm{cm}^{2}\right)$ & 0.11 & 0.06 & 0.106 & $0.04 \pm 0.024$ \\
\hline $\mathrm{Gm}$ & 4 - EFN adaxial $\left(\mathrm{cm}^{2}\right)$ & 0.18 & 0.07 & 0.017 & $0.07 \pm \mathbf{0 . 0 2 2}$ \\
\hline
\end{tabular}


Table 2 - Logistic regression of phenotypic selection on extrafloral nectaries (EFNs) of Anemopaegma album.

EFN traits were standardized to zero mean and unit variance within populations before analyses. Analyses were also performed by jackknife procedures, corroborating the phenotypic selection scenario on particular EFN descriptors. $\beta_{\text {avgrad+ }}$ was the estimated selection gradient $\beta$ as described by Janzen \& Stern (1998).

\begin{tabular}{|c|c|c|c|c|c|c|}
\hline \multirow{2}{*}{ Population } & \multirow{2}{*}{ EFN traits } & \multicolumn{4}{|c|}{ Logistic regression } & \multirow[b]{2}{*}{$\beta_{\text {avggrad }} \pm \mathrm{CI}$} \\
\hline & & $\mathrm{N}$ & $\alpha$ & SE & $p$ & \\
\hline $\mathrm{Cr}$ & 1 - EFN base & 114 & +0.31 & 0.13 & 0.021 & $\mathbf{0 . 0 7} \pm \mathbf{0 . 0 6 3}$ \\
\hline $\mathrm{Cr}$ & 2 - EFN size (mm) & 98 & -0.11 & 0.15 & 0.461 & $-0.03 \pm 0.071$ \\
\hline $\mathrm{Cr}$ & 3 - EFN abaxial $\left(\mathrm{cm}^{2}\right)$ & 114 & +0.27 & 0.13 & 0.037 & $0.06 \pm 0.061$ \\
\hline $\mathrm{Cr}$ & $4-$ EFN adaxial $\left(\mathrm{cm}^{2}\right)$ & 114 & +0.03 & 0.14 & 0.803 & $0.01 \pm 0.067$ \\
\hline $\mathrm{Gm}$ & 1 - EFN base & 112 & +0.19 & 0.13 & 0.130 & $0.05 \pm 0.062$ \\
\hline $\mathrm{Gm}$ & 2 - EFN size $(\mathrm{mm})$ & 92 & +0.09 & 0.14 & 0.544 & $0.02 \pm 0.069$ \\
\hline $\mathrm{Gm}$ & 3 - EFN abaxial $\left(\mathrm{cm}^{2}\right)$ & 112 & +0.09 & 0.13 & 0.470 & $0.02 \pm 0.062$ \\
\hline $\mathrm{Gm}$ & $4-$ EFN adaxial $\left(\mathrm{cm}^{2}\right)$ & 112 & -0.21 & 0.17 & 0.214 & $-0.05 \pm 0.081$ \\
\hline
\end{tabular}

$\mathrm{CI}=$ confidence interval. 


\section{Figures captions}

Figure 1 - Focal plant Anemopaegma album (Bignoniaceae) in the field and in the common garden. A: Shrub in the natural system (Cristália population). B: Herbivory over the margins of leaflets. C: Extrafloral nectaries clustered on the base of the abaxial side of leaflets (Arrows indicating individualized nectaries). D-E:

Inflorescences and fruits of A. album. F: Greenhouse used as a common garden, an environment with the abiotic and biotic factors controlled to cultivate offspring plants of $A$. album (Arrows indicating the water dispenser). GH: A. album seedlings. I: A. album saplings.

Figure 2 - EFN descriptors sampled on the leaves of A. Album. Character 1: abundance of EFNs calculated in the dashed square $(1 \mathrm{~cm}$ in height) on the base of abaxial side of leaflets; Character 2: size of the largest EFN on the base of abaxial side of leaflets $(1 \mathrm{~cm}$ in height); Character 3: Average abundance of EFNs in the intermediate and the apex dashed square $\left(1 \mathrm{~cm}^{2}\right.$ of area and $1 \mathrm{~cm}$ in height, respectively) on the abaxial side of leaflets; Character 4: Average abundance of EFNs in the intermediate and the apex dashed square $\left(1 \mathrm{~cm}^{2}\right.$ of area and $1 \mathrm{~cm}$ in height, respectively) on the adaxial side of leaflets.

Figure 3 - Variation of EFN abundance and size on the abaxial base of leaflets between the two populations of Anemopaegma album. A-B: On average, individuals from the Grão Mogol population had higher amounts and larger EFNs than individuals from the Cristália population. Statistical details are available in Supplementary Table S1. C: EFN size was not correlated with EFN abundance in the Cristália population (Cr), but was positively correlated in the Grão Mogol population $(\mathrm{Gm})$. ANCOVA results: $\mathrm{F}_{(3,191)}=5.95 ; \mathrm{p}_{\text {total }}<0.001 ; \mathrm{p}_{(\mathrm{EFN} * \mathrm{pop})}$ $=0.03$.

Figure 4 - Variation of the ant assemblage between the two populations of A. album. A: Ant abundance per plant was higher in Cristália than in Grão Mogol. B: Ant species ranked by the herbivore index described in Nogueira et al. (2015). The most defensive ant species were three Camponotus, one Crematogaster, one Brachymyrmex and one Pseudomyrmex species (orange circles in the online version; gray circles in the printed version). C: Proportion of plants occupied by each ant species in Cristália, in which $50 \%$ of the sampled plants were occupied by less protective ants (e.g. Cephalotes pusillus). D: Proportion of plants occupied by each ant species in Grão Mogol, in which more protective ants occupied most plants. 
Figure 5 - Phenotypic selection estimated by logistic regressions between EFN descriptors and the probability of reproduction in each A. album population: Grão Mogol (Gm, black lines) and Cristália (Cr, gray lines). EFN traits were standardized to zero mean and unit variance within populations. Significant coefficients $(p<0.05)$ were indicated by solid lines and detailed in Table 2. A and C: Directional phenotypic selection on EFNs in the Cristália population, in which plants with higher number of EFNs on average had higher probability of reproduction (solid black lines). A, B, C and D: Absence of phenotypic selection on EFN descriptors in the Grão Mogol population (dashed gray lines).

Figure 6 - Path analyses testing the defensive hypothesis mediated by EFNs against herbivores. The relationships among EFN traits, herbivory, and plant reproductive performance were in accordance with the defense hypothesis only in the Cristália population. A: Bigger EFNs (and less evident: higher EFN abundance) decreases herbivory; and subsequently, higher proportion of foliar herbivory decreases plant reproduction (Cristália). B: A lack of a direct relationship among variables in the Grão Mogol population, a pattern not predicted by the defensive hypothesis. Solid and dashed lines indicate standardized coefficients with $\mathrm{p}<0.05$ and $p<0.08$, respectively. Light gray lines indicate standardized coefficients with $p>0.08$. Statistics of each path coefficient are detailed in the Supplementary Table S3 and Supplementary Figure S2. 
Figure 1

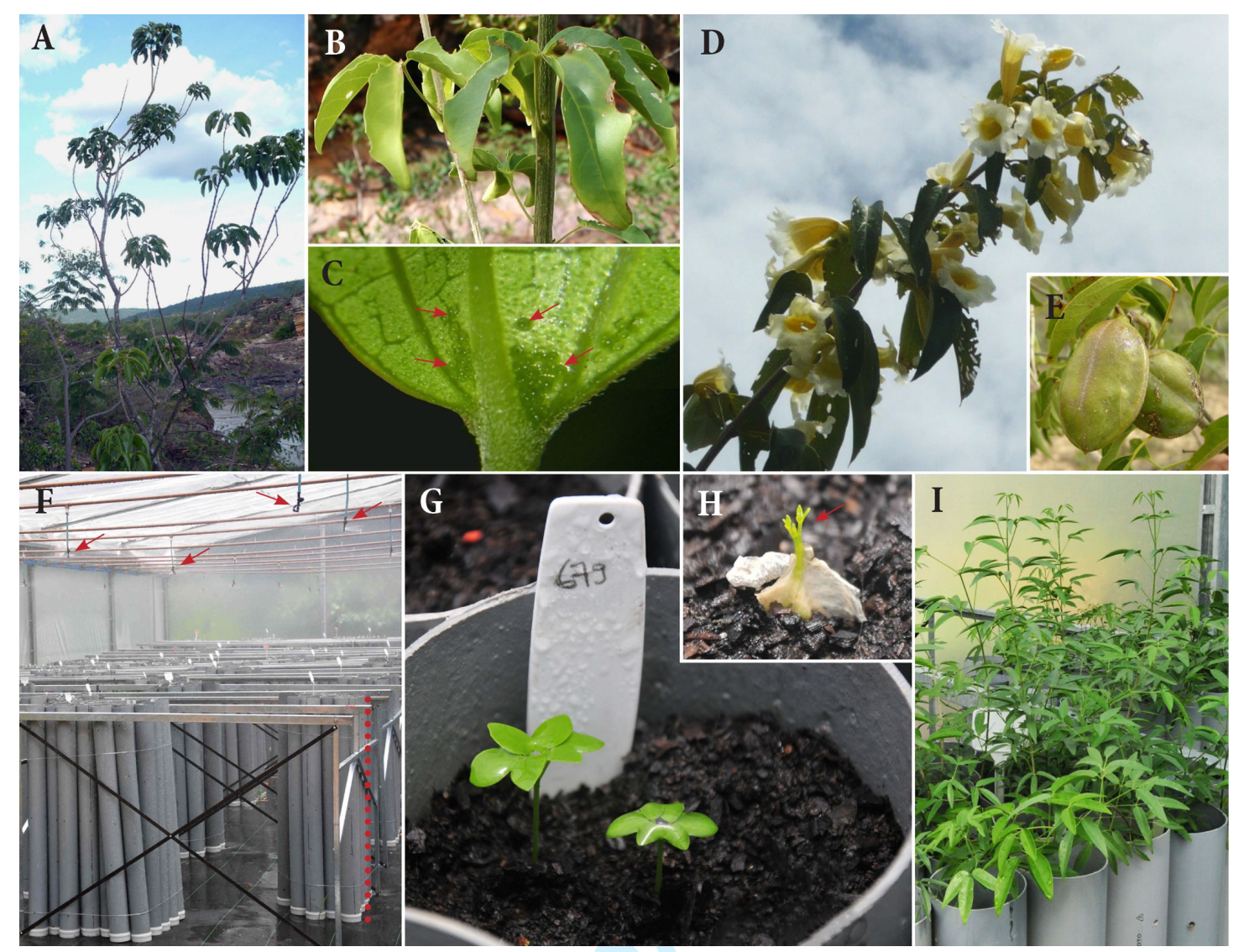


Figure 2
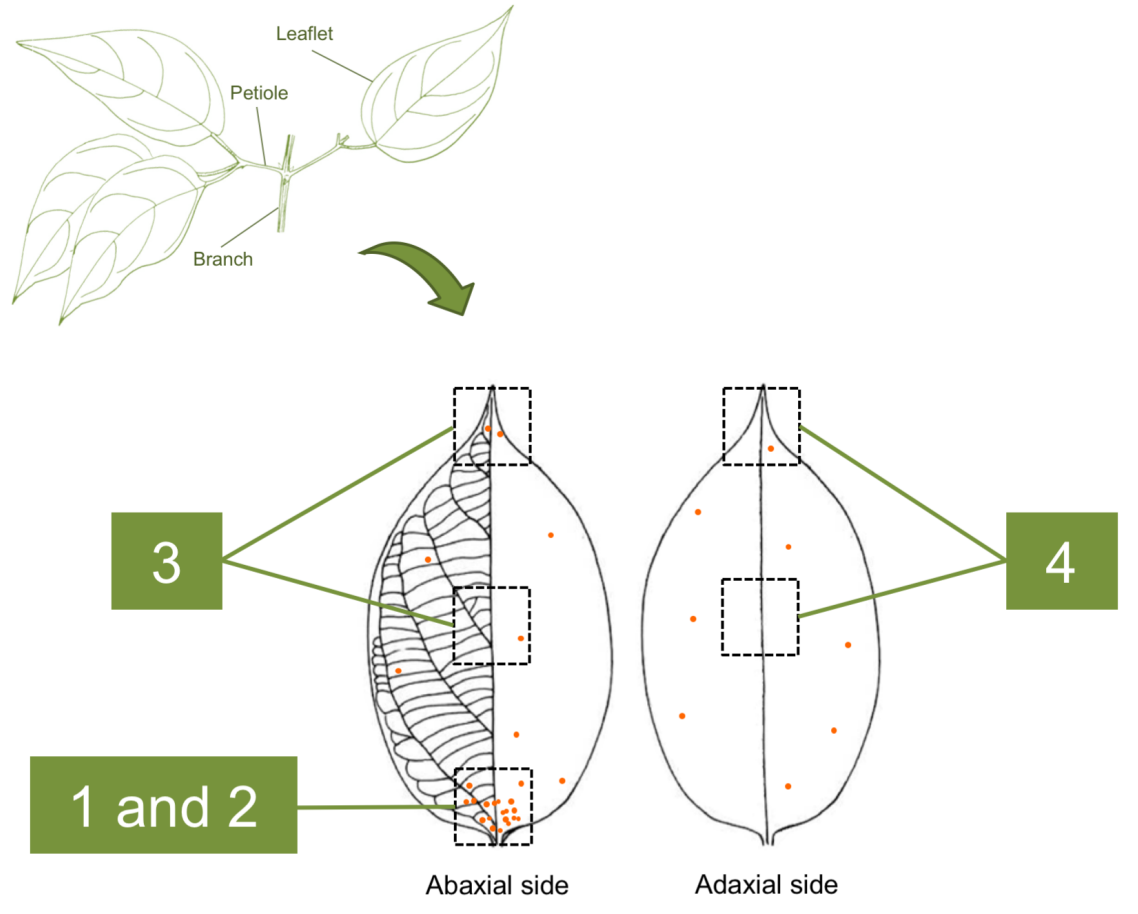
Figure 3
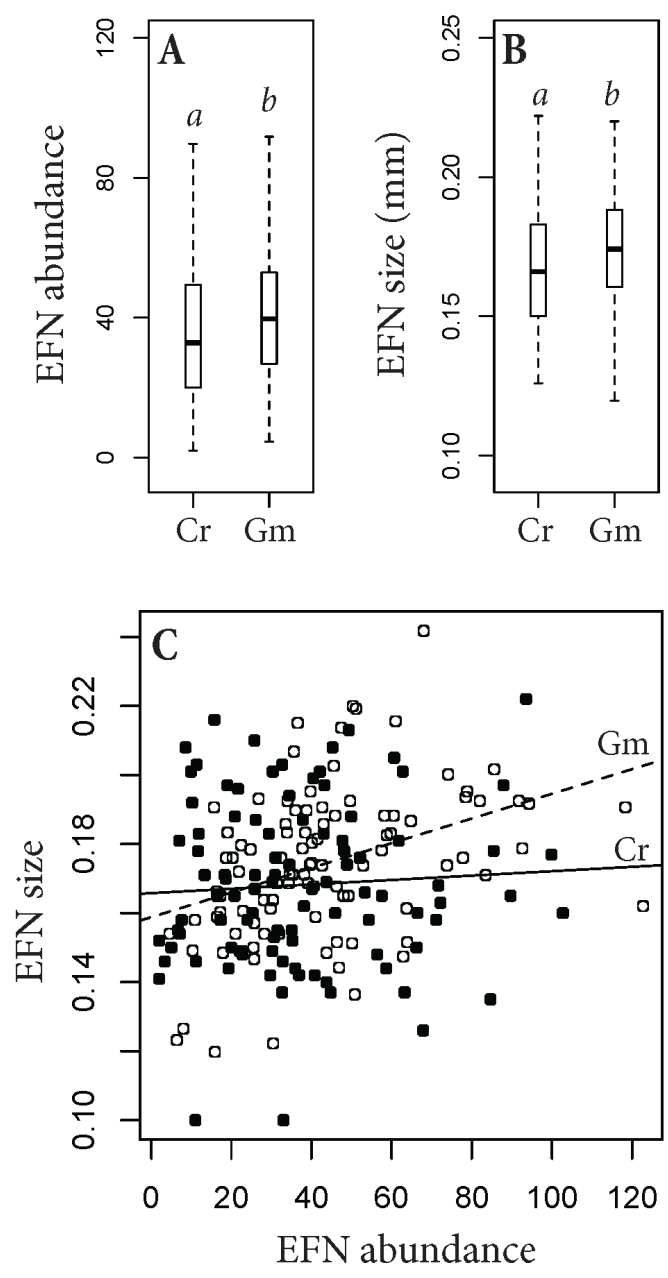

https://mc06.manuscriptcentral.com/botany-pubs 
Figure 4
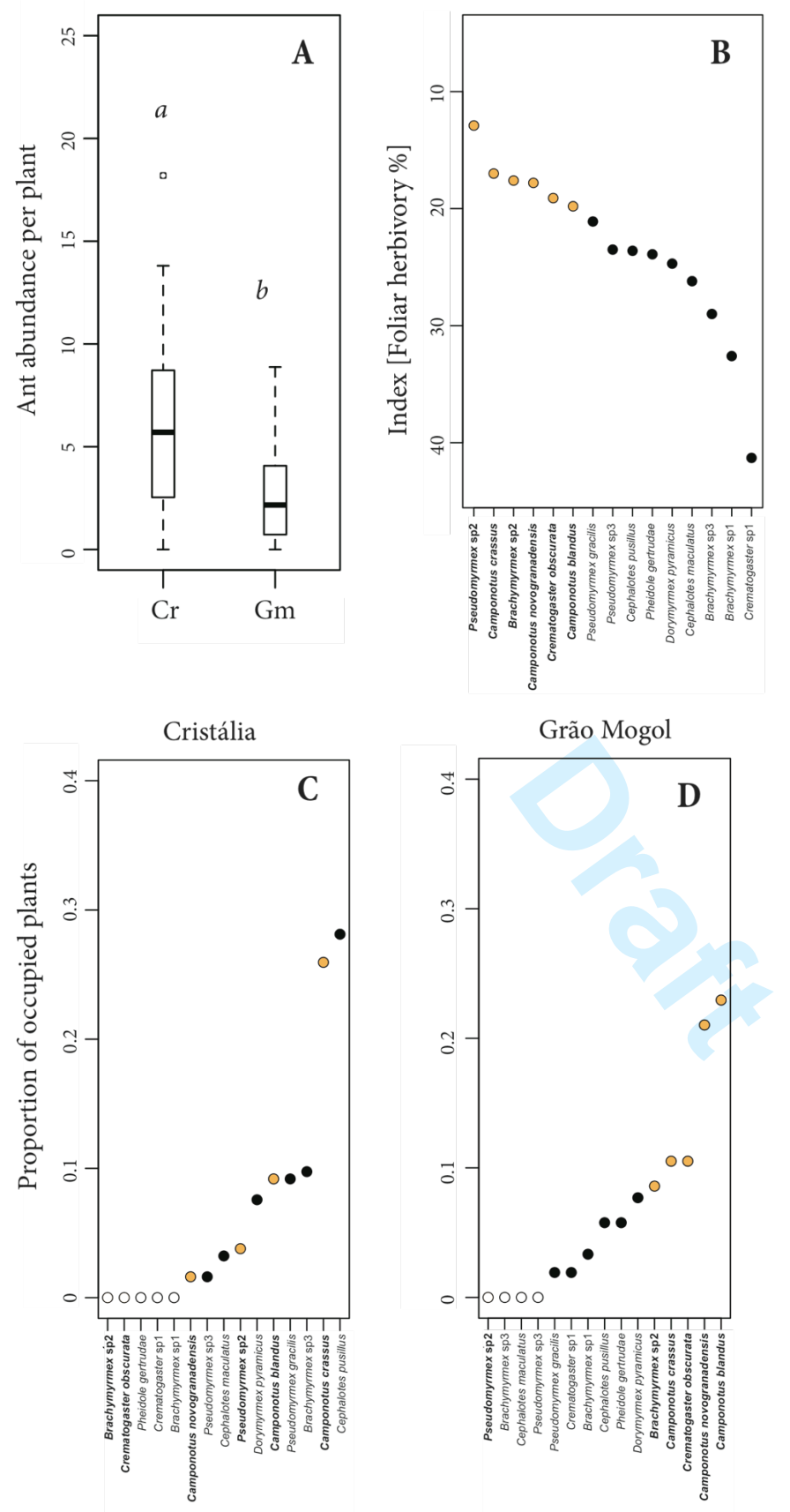
Figure 5

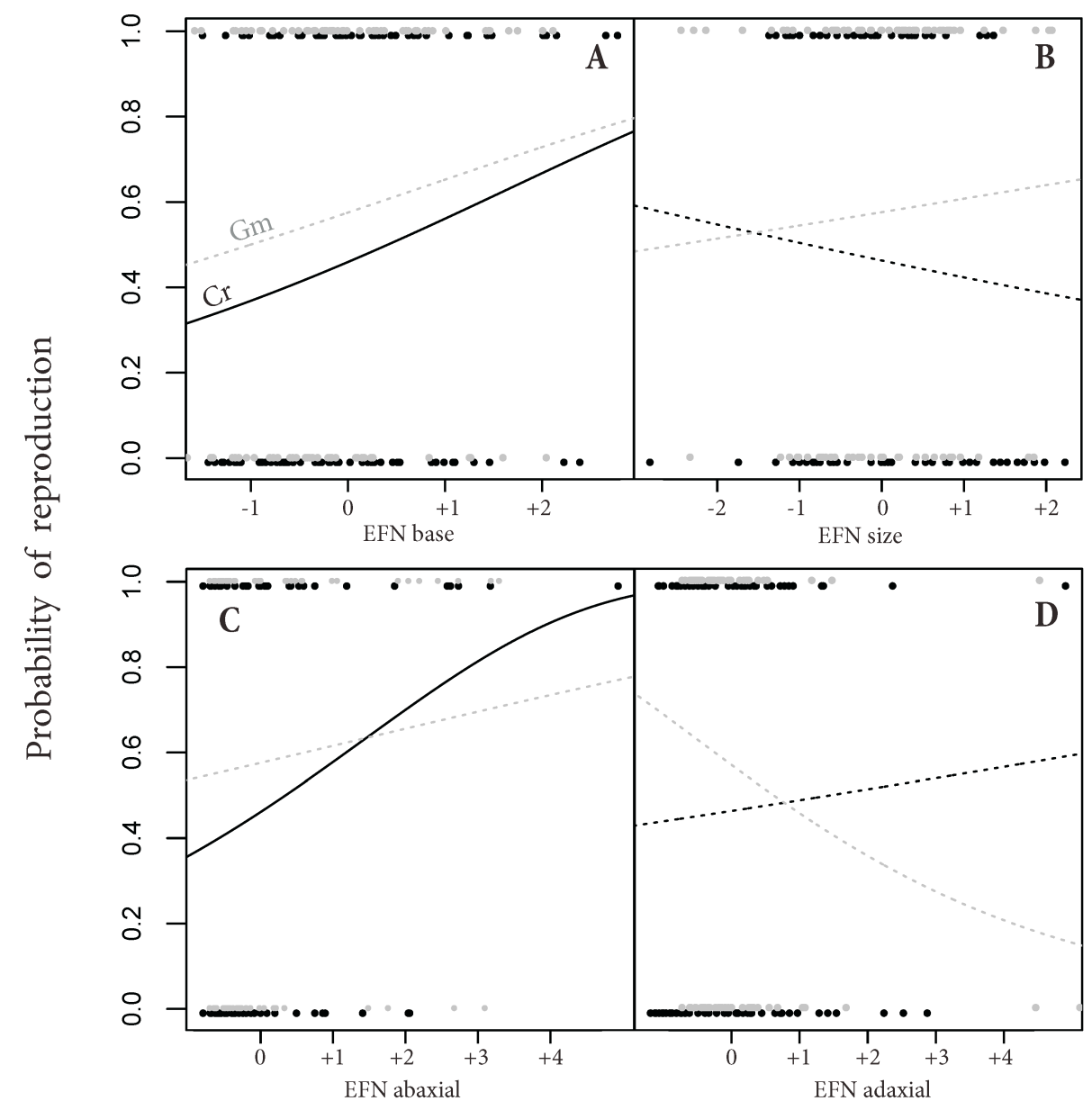




\section{Figure 6}

\section{Cristália population}

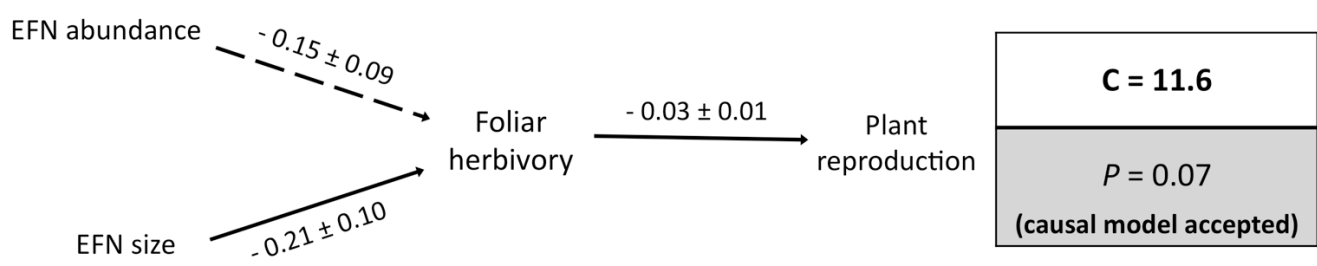

\section{Grão Mogol population}

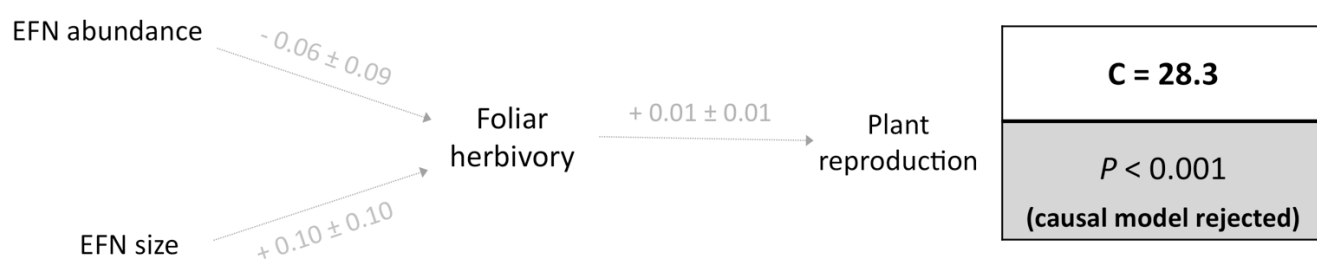

